\title{
INNOVATION AND IMPROVEMENT Warm Handoffs: a Novel Strategy to Improve End-of-Rotation Care Transitions
}

\author{
Harry S. Saag, M.D' , Jingjing Chen, A.B ${ }^{2}$, Joshua L. Denson, M.D. ${ }^{3}$, Simon Jones, Ph.D4, \\ Leora Horwitz, M.D., M.H.S ${ }^{7,4}$, and Patrick M. Cocks, M.D. ${ }^{7}$
}

\begin{abstract}
'Department of General Internal Medicine and Clinical Innovation, NYU Langone Medical Center and NYU School of Medicine, New York, NY, USA; ${ }^{2}$ NYU School of Medicine, New York, NY, USA; ${ }^{3}$ Division of Pulmonary Sciences and Critical Care, University of Colorado School of Medicine, Denver, CO, USA; ${ }^{4}$ Department of Population Health, NYU School of Medicine, New York, NY, USA.
\end{abstract}

BACKGROUND: Hospitalized medical patients undergoing transition of care by house staff teams at the end of a ward rotation are associated with an increased risk of mortality, yet best practices surrounding this transition are lacking. AIM: To assess the impact of a warm handoff protocol for end-of-rotation care transitions.

SETTING: A large, university-based internal medicine residency using three different training sites.

PARTICIPANTS: PGY-2 and PGY-3 internal medicine residents.

PROGRAM DESCRIPTION: Implementation of a warm handoff protocol whereby the incoming and outgoing residents meet at the hospital to sign out in-person and jointly round at the bedside on sicker patients using a checklist. PROGRAM EVALUATION: An eight-question survey completed by 60 of 99 eligible residents demonstrated that $85 \%$ of residents perceived warm handoffs to be safer for patients $(p<0.001)$, while 98\% felt warm handoffs improved their knowledge and comfort level of patients on day 1 of an inpatient rotation $(p<0.001)$ as compared to prior handoff techniques. Finally, 88\% felt warm handoffs were worthwhile despite requiring additional time $(p<0.001)$.

DISCUSSION: A warm handoff protocol represents a novel strategy to potentially mitigate the known risks associated with end-of-rotation care transitions. Additional studies analyzing patient outcomes will be needed to assess the impact of this strategy.

KEY WORDS: care transitions; patient safety; medical education-graduate. J Gen Intern Med 33(1):116-9

DOI: $10.1007 / \mathrm{s} 11606-017-4145-4$

(C) Society of General Internal Medicine 2017

\section{INTRODUCTION}

Transitions in care, also termed handoffs, occur when the care of a hospitalized patient is transferred between providers. Shift-to-shift handoffs, which can occur daily such as with overnight coverage or the end of a shift, are linked to delays in diagnosis, increased diagnostic testing, and adverse outcomes. $^{1,2}$ Evidence suggests implementation of structured handoff reform measures may considerably improve patient

Received April 3, 2017

Revised July 5, 2017

Accepted July 17, 2017

Published online August 14, 2017 safety during this transition. ${ }^{3-5}$ However, adverse consequences of end-of-rotation care transition, a more permanent transfer of information occurring when a physician transfers an entire list of patients, often 10-20, to a new oncoming provider, have been less well defined. ${ }^{6,7}$ Increasing evidence suggests this type of transition is associated with higher mortality, ${ }^{8,9}$ yet little standardization exists on best practices to safely perform this difficult and complicated process.

Strategies that improve end-of-rotation handoffs are urgently needed to address this potentially serious gap in clinical care. Given that failures in communication are a leading cause of adverse healthcare events, ${ }^{10}$ Solet et al. have suggested "unambiguous, face-to-face communication" between providers at the bedside as a means to ensure effective handoffs. We have defined such bedside rounding and transfer of care as "warm handoffs." While warm handoffs have been studied and recommended as a quality improvement strategy in nursing literature, ${ }^{11-13}$ its impact on resident physician end-ofrotation transitions is unknown.

Beginning July 2015, the New York University (NYU) Internal Medicine Residency Program implemented a standardized end-of-rotation transition in care protocol utilizing warm handoffs for resident physicians transferring care at the end of their rotations. We hypothesized this new model would decrease communication breakdowns and increase the incoming residents' knowledge and confidence to assume the care of their patients from day 1 compared to written or verbal sign out alone.

\section{SETTING AND PARTICIPANTS}

The NYU School of Medicine Internal Medicine Residency is a large university-affiliated residency program in New York City with 168 trainees during the 2015-2016 academic year. House staff rotate at three diverse inpatient training sites: (1) NYU Langone Medical Center-Tisch Hospital, a universitybased academic medical center; (2) Bellevue Hospital, a large public hospital and member of New York City Health and Hospitals Corporation; (3) Veterans Affairs New York Harbor Healthcare System, Manhattan Campus. Eligible participants included all post-graduate year (PGY) 2 and 3 residents. 


\section{PROGRAM DESCRIPTION}

Prior to July 2015, outgoing residents handed off their inpatient service prior to service change in a nonstandardized fashion, typically providing an e-mailed written or telephonic verbal sign out. While residents received training on appropriate shift-to-shift handoffs using IPASS ${ }^{4}$ no formal training was done for end-of-service transitions.

In July 2015, a 1-h formal training session was given to all PGY-2/3 residents on how to perform warm handoffs. This training included reviewing evidence showing that patients are at an increased risk for mortality at the time of team transition ${ }^{8}$ as well as program expectations of how and when warm handoffs should be performed. Beginning July 2015, it was expected that the incoming and outgoing PGY-2/3 resident would jointly perform a warm handoff on the day prior to resident switch day for all inpatient medical ward services across all three hospital sites. Interns were not expected to perform warm handoffs nor residents when rotating on and off ICU teams. Otherwise, warm handoffs were the expectation of the program for all other service changes. The warm handoff was expected to include the incoming and outgoing residents meeting face to face at the hospital to transition care including discussing patient's past medical history, history of presenting illness, hospital course, assessment, and plan. Further, residents were expected to jointly round on the sickest patients as determined by the opinion of the outgoing resident. A bedside rounding checklist (Text Box 1) was developed internally to guide the structure of joint bedside rounding to maximize impact and efficiency focusing on elements that are best conveyed while at the patient's bedside such as physical examination findings. An email was sent by a Chief Resident before switch days reminding residents to perform warm handoffs on the day prior to switch day along with a bedside rounding checklist that was introduced at the formal training session (Text Box 1). All PGY-2/3 residents rotate services every $2-4$ weeks on the same day of the week.

Text Box 1: Bedside Rounding Checklist.

\begin{tabular}{l}
\hline \hline A. New symptoms overnight \\
B. Same day discharge plans, complicated discharge plans, social \\
barriers to discharge or care \\
C. Physical examination with attention to: \\
i. Invasive equipment (Foley, CVC, rectal tube, vent, PICC, PEG) \\
ii. Pain \\
iii. Altered mental status \\
iv. Neuro deficits \\
v. Volume status \\
vi. Skin/cellulitis findings \\
vii. Wounds \\
viii. Acute abdomen \\
ix. Post-procedure \\
x. Increased work of breathing or wheezing \\
Abbreviations legend: \\
CVC: central venous catheter \\
Vent: ventilator \\
PICC: peripherally inserted central catheter \\
PEG: percutaneous endoscopic gastrostomy tube \\
\hline
\end{tabular}

\section{PROGRAM EVALUATION}

The warm-handoff approach was studied quantitatively using surveys and qualitatively using face-to-face semi-structured open-ended interviews. The mixed-method approach evaluated the implementation rates, impact on perceived patient safety, and resident satisfaction. Qualitative interviews allowed exploration of perceived strengths and weaknesses of the warm-handoff approach and identified possible areas for future improvement.

An eight-question voluntary, anonymous, internet-based survey developed internally was sent to all 99 PGY $2 / 3$ residents in April 2016, roughly 10 months following the start of the intervention. Six residents were randomly selected to participate in face-to-face standardized open-ended interviews that helped to identify underlying factors driving perceptions of patient safety and resident satisfaction. No compensation or incentives were provided for participation. All quantitative data were analyzed using SAS Enterprise Guide 6.1 and SAS 9.4 (SAS Institute Inc., Cary NC). Chi-squared test and Fisher's exact test were used to analyze associations of categorical variables. Ninety-five percent confidence intervals were calculated using binomial confidence intervals.

\section{SURVEY FINDINGS}

Sixty of the eligible 99 PGY 2/3 residents completed the survey $(60.6 \%)$. Survey data for each individual survey question can be seen in Table 1 . Survey data showed that at baseline, only $5 \%$ of residents signed out in person with $95 \%$ using a combination of written and/or verbal sign out to transition care. Following our intervention, $92 \%$ of surveyed residents answered that they were performing warm handoffs "half the time" or "almost always" as compared to written or verbal sign out.

The strengths of the warm handoff initiative were demonstrated by an overwhelmingly positive response on a number of survey items. Following the intervention, $98 \%$ (95\% CI, 95\%$100 \%$ ) acknowledged end-of-rotation transitions were a vulnerable time for patients, and $85 \%$ (95\% CI, 75\%-93\%) of residents perceived warm handoffs to be safer for patients than written/verbal handoffs. In addition, 87\% (95\% CI, 78\%-95\%) of residents also reported an improved knowledge and comfort level of their patients on day 1 of a new rotation. Although $75 \%$ (95\% CI, 58\%-85\%) of residents reported spending an extra hour or more to perform a warm handoff, $88 \%$ (95\% CI, $80 \%-$ 95\%) felt that warm handoffs were a worthwhile use of their time. Perception of improved patient safety was associated with residents' improved sense of preparedness $(p<0.001)$ as compared to prior handoff techniques and residents' perception of warm handoffs as a worthwhile intervention $(p<0.001)$.

\section{QUALITATIVE FINDINGS}

During semi-structured interviews, all participants $(n=6)$ cited the ability to see patients in person as a major advantage of 
Table 1 Survey Results Assessing Warm Handoffs

\begin{tabular}{|c|c|c|c|}
\hline & $\begin{array}{l}\text { Number of } \\
\text { responses }\end{array}$ & $\begin{array}{l}\% \\
\text { response }\end{array}$ & $\begin{array}{l}\text { Nominal 95\% } \\
\text { confidence interva } \\
\text { (CI) }\end{array}$ \\
\hline \multicolumn{4}{|c|}{ End-of-rotation transitions a vulnerable time for patients } \\
\hline No & 1 & $2 \%$ & $(0 \%-5 \%)$ \\
\hline Yes & 59 & $98 \%$ & $(95 \%-100 \%)$ \\
\hline \multicolumn{4}{|c|}{ Sign out methods before warm handoff } \\
\hline $\begin{array}{l}\text { In person at } \\
\text { the bedside }\end{array}$ & 2 & $3 \%$ & $(0 \%-8 \%)$ \\
\hline $\begin{array}{l}\text { In person at } \\
\text { the hospital }\end{array}$ & 1 & $2 \%$ & $(0 \%-5 \%)$ \\
\hline Verbal & 11 & $18 \%$ & $(5 \% 28 \%)$ \\
\hline $\begin{array}{l}\text { Verbal and } \\
\text { written }\end{array}$ & 40 & $67 \%$ & $(48 \%-78 \%)$ \\
\hline Written & 6 & $10 \%$ & $(2 \%-18 \%)$ \\
\hline \multicolumn{4}{|c|}{ Number of patients rounded on during warm handoff } \\
\hline $0-2$ & 3 & $5 \%$ & $(0 \%-12 \%)$ \\
\hline $3-6$ & 23 & $38 \%$ & $(22 \%-50 \%)$ \\
\hline $7-10$ & 21 & $35 \%$ & $(18 \%-47 \%)$ \\
\hline$>10$ & 13 & $22 \%$ & $(8 \%-32 \%)$ \\
\hline \multicolumn{4}{|c|}{ Current frequency of participation in warm handoffs } \\
\hline Almost never & 5 & $8 \%$ & $(2 \%-17 \%)$ \\
\hline Half the time & 23 & $38 \%$ & $(27 \%-50 \%)$ \\
\hline Almost & 32 & $53 \%$ & $(40 \%-67 \%)$ \\
\hline \multirow{2}{*}{\multicolumn{4}{|c|}{$\begin{array}{l}\text { always } \\
\text { Warm handoffs safer for patients compared to written/verbal sign out }\end{array}$}} \\
\hline & & & \\
\hline No & 9 & $15 \%$ & $(7 \%-25 \%)$ \\
\hline Yes & 51 & $85 \%$ & $(75 \%-93 \%)$ \\
\hline \multicolumn{4}{|c|}{$\begin{array}{l}\text { Improved knowledge and comfort level with patients using warm } \\
\text { handoffs }\end{array}$} \\
\hline No & 7 & $12 \%$ & $(5 \%-20 \%)$ \\
\hline Yes & 52 & $87 \%$ & $(78 \%-95 \%)$ \\
\hline (Missing) & 1 & $2 \%$ & $(0 \%-5 \%)$ \\
\hline \multirow{2}{*}{\multicolumn{4}{|c|}{$\begin{array}{l}\text { Amount of extra time required for warm handoffs vs. prior sign out } \\
\text { No additional } 3 \\
\begin{array}{l}5 \% \\
\text { time }\end{array}\end{array}$}} \\
\hline & & & \\
\hline 30 min extra & 12 & $20 \%$ & $(7 \%-30 \%)$ \\
\hline $1 \mathrm{~h}$ extra & 30 & $50 \%$ & $(32 \%-63 \%)$ \\
\hline More than 1 & 15 & $25 \%$ & $(10 \%-37 \%)$ \\
\hline & & & \\
\hline \multicolumn{4}{|c|}{$\begin{array}{l}\text { Assuming warm handoffs take extra time, warm handoffs still } \\
\text { worthwhile }\end{array}$} \\
\hline No & 7 & $12 \%$ & $(5 \%-20 \%)$ \\
\hline Yes & 53 & $88 \%$ & $(80 \%-95 \%)$ \\
\hline
\end{tabular}

warm handoffs. Several residents spoke of the importance of seeing a baseline examination for stroke patients, encephalopathy patients, or medically complex patients. Bedside rounding also helped remind outgoing residents of important findings that would not have otherwise been communicated. Half of the respondents reported face-to-face interaction between providers decreased misinformation and improved communication. Examples included use of non-verbal language cues and gestures, active participation (versus passively receiving email), and real-time question and response.

Disadvantages discussed included interruptions during warm handoffs, as warm handoffs occur during and not after the work day ends. Time limitations also prevented residents from conducting a bedside round on every patient.

\section{DISCUSSION}

End-of-rotation care transitions are increasingly recognized as a vulnerable time for hospitalized medical patients. While these patients may have $12-34 \%$ higher adjusted odds of hospital death when exposed to this transition, ${ }^{8,9}$ few hospital and medical education systems have standardized a strategy to define best practices for transitioning care during these inopportune times despite recommendations from The Joint Commission and The Accreditation Council for Graduate Medical Education to train residents on communications surrounding shift-to-shift handoff. ${ }^{14}$ In this single institution survey study, 98\% of surveyed resident physicians felt end-of-rotation transitions placed patients at risk. Warm handoffs at the time of service change between incoming and outgoing upper-level residents represent an innovative approach that may lessen this risk. This intervention clearly changed the way in which residents handed off their service as evidenced by only $5 \%$ of surveyed residents indicating they sign out in person at the hospital or bedside at baseline compared with greater than $90 \%$ now performing in-person warm handoffs at least half the time following our intervention.

Further, residents perceive warm handoffs to be safer for patients as compared to prior sign out techniques with greater than $85 \%$ of respondents citing improved knowledge and comfort level with patients when coming on to a new service using warm handoff. This perceived increase in safety and knowledge of patients following service change is consistent with prior research on shift-to-shift handoff, which has shown decreased adverse events when using a standardized, structured handoff technique. ${ }^{4}$

Qualitative data confirm that the ability to see patients in person is one of the most important aspects contributing to an increased sense of safety and knowledge of the patients, particularly when a patient has a notable physical examination finding or other pertinent finding as noted on the warm handoff rounding checklist (Text Box 1).

Most importantly, residents acknowledge that performing a warm handoff takes additional time compared to verbal or written sign out, yet they believe there is value in spending the extra time it takes to perform a warm handoff. This is particularly striking given that residents work significant hours at baseline and therefore would be less likely to voluntarily devote more of their time toward work than is absolutely required.

There were several limitations to our study that must be acknowledged. First, we were unable to collect survey data on all eligible PGY-2/3's; however, we were able to collect data on over half of all eligible house staff. Second, no monitoring or supervision of warm handoffs took place to confirm the accuracy of house staff-reported answers on how often they performed warm handoffs or how many patients they truly rounded on at the bedside. Further, it is unknown how standardized or similar the warm handoffs were between residents in terms of bedside rounding techniques or information transfer. Despite this, there is little reason to suspect house staff would overestimate their participation or how much time they spent performing warm handoffs. Third, our study was retrospective in nature and looks at only a single residency program. Additionally, both our bedside rounding checklist and survey were developed internally without validation, although 
development was guided by resident feedback, previously reported qualitative studies, ${ }^{15}$ and from other types of handoff literature. ${ }^{2,11-13}$ Lastly, our study does not look at patient outcomes; thus, there is no way to measure what impact, if any, warm handoffs may have on patient outcomes, which will be important to estimate in future studies.

In conclusion, our study represents a novel strategy that may reduce the risk associated with end-of-rotation service changes. While it is uncertain whether warm handoffs affect patient outcomes, it is clear that residents perceive warm handoffs as a safer way to transition care at the time of service change and rate the exercise as a valuable use of their time. Further studies will need to assess whether this perceived increase in safety translates to safer outcomes for patients.

Corresponding Author: Harry S. Saag, M.D; Department of General Internal Medicine and Clinical InnovationNYU Langone Medical Center and NYU School of Medicine, New York, NY, USA (e-mail: Harry.Saag@nyumc.org).

\section{Compliance with Ethical Standards:}

Conflict of Interest: The authors declare no conflicts of interest.

\section{REFERENCES}

1. Horwitz LI, Moin T, Krumholz HM, Wang L, Bradley EH. Consequences of inadequate sign-out for patient care. Arch Intern Med. 2008;168:1755-1760.
2. Riesenberg LA, et al. Residents' and attending physicians' handoffs: a systematic review of the literature. Acad Med J Assoc Am Med Coll. 2009;84:1775-1787.

3. Starmer AJ, et al. Rates of medical errors and preventable adverse events among hospitalized children following implementation of a resident handoff bundle. JAMA. 2013;310:2262-2270.

4. Starmer AJ, et al. Changes in medical errors after implementation of a handoff program. N Engl J Med. 2014;371:1803-1812.

5. Horwitz LI, Moin T, Krumholz HM, Wang L, Bradley EH. What are covering doctors told about their patients? Analysis of sign-out among internal medicine house staff. Qual Saf Health Care. 2009; 18:248-255.

6. Rich EC, Gifford G, Dowd B. The effects of scheduled intern rotation on the cost and quality of teaching hospital care. Eval Health Prof 1994;17:259-272.

7. Smith JP, et al. Effects of end-of-month admission on length of stay and quality of care among inpatients with myocardial infarction. Am J Med. 2002;113:288-293.

8. Denson JL, McCarty M, Fang Y, Uppal A, Evans L. Increased mortality rates during resident handoff periods and the effect of ACGME duty hour regulations. Am J Med. 2015;128:994-1000.

9. Denson JL, et al. Association between end-of-rotation resident transition in care and mortality among hospitalized patients. JAMA. 2016;316:2204-2213.

10. Streitenberger $\mathbf{K}$, Breen-Reid $\mathbf{K}$, Harris $\mathbf{C}$. Handoffs in care-can we make them safer? Pediatr Clin N Am. 2006;53:1185-1195

11. Chaboyer W, et al. Bedside handover: quality improvement strategy to 'transform care at the bedside'. J Nurs Care Qual. 2009;24:136-142.

12. Cairns LL, Dudjak LA, Hoffmann RL, Lorenz HL. Utilizing bedside shift report to improve the effectiveness of shift handoff. J Nurs Adm. 2013;43:160-165.

13. Taylor J. Improving patient safety and satisfaction with standardized bedside handoff and walking rounds. Clin J Oncol Nurs. 2015;19:414-416.

14. Sehgal N. Annual Perspective 2014: Handoffs and Transitions. Agency for Healthcare Research and Quality Patient Safety Network. 2015. http:// psnet.ahrq.gov/perspective.aspx?perspectiveID=170.

15. Bernabeo EC, Holtman MC, Ginsburg S, Rosenbaum JR, Holmboe ES. Lost in transition: the experience and impact of frequent changes in the inpatient learning environment. Acad Med. 2011;86:591-598. 